\title{
A new protocol for pyrrole synthesis by a combination of ring- closing metathesis and in situ oxidative aromatization
}

\author{
Nicolai Dieltiens, ${ }^{a}$ Christian V. Stevens, ${ }^{* a}$ Bart Allaert, ${ }^{b}$ and Francis Verpoort ${ }^{b}$ \\ ${ }^{a}$ Research group SynBioC, Department of Organic Chemistry, Faculty of Bioscience \\ Engineering, Ghent University, Coupure links 653, B-9000 Ghent, Belgium \\ ${ }^{b}$ Division of Organometallics and Catalysis, Department of Inorganic and Physical Chemistry, \\ Ghent University, Krijgslaan 281 (S-3), B-9000 Ghent, Belgium \\ E-mail: chris.stevens@ugent.be
}

(received 08 Dec 04; accepted 07 Jan 05; published on the web 12 Jan 05)

\begin{abstract}
A new and straightforward protocol for pyrrole synthesis is developed by a unique one-pot combination of ring-closing metathesis and in situ oxidative aromatization by a quinone.
\end{abstract}

Keywords: Pyrrole synthesis, ring-closing metathesis, oxidative aromatization, transfer hydrogenation

\section{Introduction}

The occurrence of the pyrrole nucleus in many natural and synthetic biologically active compounds continues to contribute to the development of new synthetic methodologies towards this important heterocycle. ${ }^{1}$ Recently we reported on the development of a new catalytic tandem comprising the second generation Grubbs' catalyst and $\mathrm{RuCl}_{3} \times \mathrm{H}_{2} \mathrm{O}$. ${ }^{2}$ This catalytic couple is able to convert diallylamines 1 into the corresponding pyrroles 2 (Scheme 1). A weak point of this method is the long reaction time (over $12 \mathrm{~h}$ for complete conversion). In this paper we report an improved methodology using a strong hydrogen acceptor (tetrachloro-1,4-benzoquinone) to speed up the aromatization process.

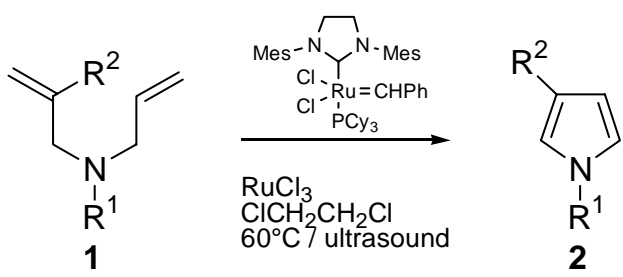

Scheme 1. Pyrrole synthesis using the second generation Grubbs' catalyst in combination with $\mathrm{RuCl}_{3} \times \mathrm{H}_{2} \mathrm{O}$. 


\section{Results and Discussion}

In an effort to speed up the dehydrogenation process, the possibility of adding a strong hydrogen acceptor was explored. As a first choice, 1 equivalent of DDQ 3 (Figure 1) was added to the reaction mixture since it is known that pyrrolines can be converted to the corresponding pyrroles by DDQ. ${ }^{3}$ Analyzing our first results, no ring-closing metathesis could be observed, suggesting that the second generation Grubbs' catalyst and DDQ are incompatible. Literature study however, revealed that a combination of ring-closing metathesis and oxidative aromatization was recently reported as a new protocol for benzoannulation. ${ }^{4}$ Although this is a one-pot reaction, the DDQ was added after metathesis, thus avoiding catalyst inactivation. In a quest for quinones which do not react with the second generation Grubbs' catalyst, our attention turned to tetramethyl-1,4-benzoquinone 4 (duroquinone) and tetrachloro-1,4-benzoquinone 5 (chloranil) as strong hydrogen acceptors (Figure 1). These quinones have been evaluated before as hydrogen acceptor in combination with $\mathrm{RuCl}_{3} \times \mathrm{H}_{2} \mathrm{O}{ }^{5}$
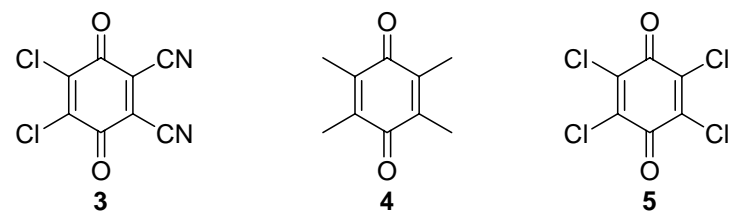

Figure 1. The evaluated different hydrogen acceptors: DDQ (3), duroquinone (4) and chloranil (5).

Upon evaluation of 4, we were pleased to find that ring-closing metathesis was not inhibited, however, the rate of aromatization was not significantly influenced. Finally, adding tetrachloro1,4-benzoquinone together with the second generation Grubbs' catalyst resulted in the complete conversion of the diallylamine to the corresponding pyrrole within 2 hours. It was even observed that $\mathrm{RuCl}_{3} \times \mathrm{H}_{2} \mathrm{O}$ is not necessary in this conversion. This might suggest that either the metathesis catalyst, or decomposition compounds thereof, catalyze the hydrogen transfer or that hydrogen is directly transferred from donor to acceptor. A possible mechanism for this reaction sequence (in accordance to the mechanism of the reaction of Hantzsch esters to pyridines ${ }^{6}$ ) is outlined in Scheme 2. After ring-closing metathesis, the electron lone pair on $\mathrm{N}$ of the intermediate 3-pyrroline 6 initiates the aromatization by expelling a hydride which immediately reacts with 5. This assumption is consistent with the observation that diallylamines with strong electronwithdrawing groups on $\mathrm{N}$ do not aromatize. Possibly both donor and acceptor are coordinated to the transition metal center in this step, thus facilitating the H-transfer. In the next step, the intermediate iminium-ion 7 loses another proton and aromatizes to the pyrrole $\mathbf{2}$. In a final step, a proton-shift converts 9 to hydroquinone $\mathbf{1 0}$. 


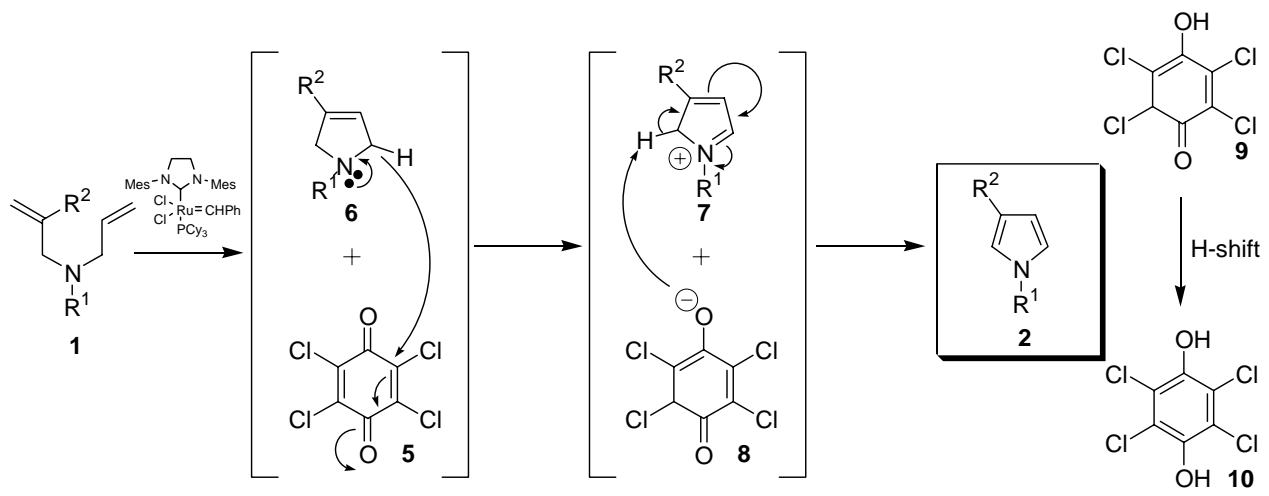

Scheme 2. Proposed mechanism for ring-closing metathesis with in situ oxidative aromatization.

Following this methodology, a number of pyrroles were synthesized with very high conversion compared to the $\mathrm{RuCl}_{3}$ system published before (Table 1).

Table 1. Synthesis of different pyrroles by a combination of second generation Grubbs' catalyst and tetrachloro-1,4-benzoquinone starting from diallylamines of type $\mathbf{1}$

\begin{tabular}{llll}
\hline Entry No. & $\mathrm{R}^{1}$ & $\mathrm{R}^{2}$ & ${\text { Conversion }(\%)^{\mathrm{a}}}^{\mathrm{a}}$ \\
\hline 1 & $\mathrm{Bn}$ & $\mathrm{H}$ & 100 \\
2 & $\mathrm{Bn}$ & $\mathrm{CH}_{3}$ & 93 \\
3 & $\mathrm{Bn}$ & $\mathrm{CH}_{2}$ morpholine & $90^{\mathrm{b}}$ \\
4 & $\mathrm{CH}_{2} \mathrm{COOMe}$ & $\mathrm{H}$ & 96 \\
5 & $\mathrm{CH}_{2} \mathrm{P}(\mathrm{O})(\mathrm{OEt})_{2}$ & $\mathrm{H}$ & 95 \\
6 & o-methoxyPh & $\mathrm{H}$ & 100 \\
7 & $\mathrm{Bn}$ & $\mathrm{Cl}$ & $0^{\mathrm{c}}$ \\
\hline
\end{tabular}

${ }^{a}$ Determined by the ${ }^{1} \mathrm{H}-\mathrm{NMR}$ spectrum of the crude reaction mixture. ${ }^{\mathrm{b}}$ The pyrrole broke down on the silica column during purification. ${ }^{\mathrm{c}}$ Only dimer could be isolated.

The yields of the purification of the pyrroles were previously described and a significant drop in yield is observed during flash chromatography (usually around $20 \%)^{2}$

For the moment the main weakness of this methodology is the activity of the metathesis catalyst. Highly substituted pyrroles for example are for the moment still out of reach. This is exemplified by entry 7 were the attempted synthesis of a 3-chloro substituted pyrrole failed, only dimer could be isolated from the reaction mixture.

\section{Conclusions}

The combination of the second generation Grubbs' catalyst and chloranil (tetrachloro-1,4benzoquinone) provides a easy way to convert diallylamines to the corresponding pyrroles. It 
also proves that ring-closing metathesis can be performed in the presence of a quinone which opens the way to the one-pot synthesis of other aromatic systems.

\section{Experimental Section}

General Procedures. All ${ }^{1} \mathrm{H}-\mathrm{NMR},{ }^{13} \mathrm{C}-\mathrm{NMR}$ and ${ }^{31} \mathrm{P}-\mathrm{NMR}$ spectra were recorded on a JEOL JNM-ECP 300-spectrometer. The purity was checked by quantitative GC analysis (Agilent 6890 Series. Phase EC-5, length $30 \mathrm{~m}$, ID $0.25 \mathrm{~mm}$, film thickness $0.25 \mu \mathrm{m}$, carrier gas $\mathrm{N}_{2}$, detector gas $\mathrm{H}_{2}$ ). All mass spectra were recorded by GC-MS coupling. A Hewlett-Packard 6890 GC Plus (carrier gas He, $1.2 \mathrm{ml} \mathrm{min}^{-1}$ ) was used coupled with a HP 5973 MSD (Mass Selective DetectorQuadrupole type, ionization EI 70eV), equipped with a CIS-4 PTV (Programmed Temperature Vaporization) injector (Gertsel), and a HP5-MS capillary column (30 x 0.25mm i.d.; coating thickness $0.25 \mu \mathrm{m})$.

\section{General procedure for pyrrole synthesis}

In a dry reaction tube, $50 \mathrm{mg}$ of diallylamine is weighed and dissolved in 1,2-dichloroethane $(0.05 \mathrm{M})$. To this solution, $5 \mathrm{~mol} \%$ of Grubbs' second generation catalyst and 0.75 equivalents of tetrachloro-1,4-benzoquinone are added. The reaction tube is sealed and placed in an oil bath at $70-75^{\circ} \mathrm{C}$. After $1 \mathrm{~h}$, the tube is removed from the oil bath, opened and $5 \mathrm{~mol} \%$ of Grubbs' second generation catalyst and 0.75 equivalents of tetrachloro-1,4-benzoquinone are added for a second time. The tube is sealed and placed back in the oil bath for an additional hour. Afterwards the solvent is removed in vacuo and a ${ }^{1} \mathrm{H}-\mathrm{NMR}$ spectrum is taken to check the conversion. The crude pyrrole is coated on silica-gel and purified by flash-chromatography (with an appropriate mixture of hexanes-ethyl acetate).

1-Benzyl-1H-pyrrole ${ }^{7}$ (entry 1). ${ }^{1} \mathrm{H}$ NMR $\left(300 \mathrm{MHz}, \mathrm{CDCl}_{3}\right) \delta: 5.07\left(2 \mathrm{H}, \mathrm{s}_{,} \mathrm{CH}_{2} \mathrm{Ph}\right), 6.19(2 \mathrm{H}$, $\mathrm{t}, \mathrm{J}=2.1 \mathrm{~Hz}, \mathrm{HCCH}), 6.69(2 \mathrm{H}, \mathrm{t}, \mathrm{J}=2.1 \mathrm{~Hz}, \mathrm{HCNCH}), 7.09-7.40(5 \mathrm{H}, \mathrm{m}, \mathrm{Ph}) .{ }^{13} \mathbf{C} \mathbf{N M R}(\mathbf{7 5}$

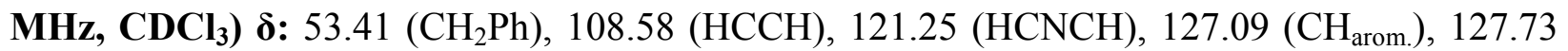
$\left(\mathrm{CH}_{\text {arom. }}\right), 128.81\left(\mathrm{CH}_{\text {arom. }}\right), 138.26\left(\mathrm{C}_{\text {arom. }}\right) . \mathrm{IR}\left(\mathrm{cm}^{-1}\right) v_{\max }: 1683$ (br). MS: $\mathrm{m} / \mathrm{z}(\%): 157\left(\mathrm{M}^{+}\right.$, 65), 91 (100), 65 (12).

1-Benzyl-3-methyl-1H-pyrrole ${ }^{8}$ (entry 2). ${ }^{1} \mathrm{H}$ NMR $\left(300 \mathrm{MHz}, \mathrm{CDCl}_{3}\right) \delta: 2.10\left(3 \mathrm{H}, \mathrm{s}, \mathrm{CH}_{3}\right)$, $4.98\left(2 \mathrm{H}, \mathrm{s}, \mathrm{CH}_{2} \mathrm{Ph}\right), 6.00(1 \mathrm{H}, \mathrm{s}, \mathrm{CH}), 6.44(1 \mathrm{H}, \mathrm{s}, \mathrm{CH}), 6.58(1 \mathrm{H}, \mathrm{s}, \mathrm{CH}), 7.21-7.36(5 \mathrm{H}, \mathrm{m}$, $\mathrm{Ph}) .{ }^{13} \mathrm{C} \mathrm{NMR}\left(75 \mathrm{MHz}, \mathrm{CDCl}_{3}\right) \delta: 12.09\left(\mathrm{CH}_{3}\right), 53.34\left(\mathrm{CH}_{2} \mathrm{Ph}\right), 109.74(\mathrm{HC}), 116.29\left(\mathrm{C}_{\mathrm{q} \text { pyrrole }}\right)$, $119.25(\mathrm{HCN}), 121.10(\mathrm{HCN}), 127.15\left(\mathrm{CH}_{\text {arom. }}\right), 127.65\left(\mathrm{CH}_{\text {arom. }}\right), 128.78\left(\mathrm{CH}_{\text {arom }}\right), 138.51$ $\left(\mathrm{C}_{\text {arom. }}\right)$. IR $\left.\left(\mathrm{cm}^{-1}\right) v_{\max }: 1498,1454\right) . \mathrm{MS}: \mathrm{m} / \mathrm{z}(\%): 171\left(\mathrm{M}^{+}, 74\right), 170$ (18), $92(10), 91$ (100), 65 (13).

Methyl 1H-pyrrol-1-yl acetate (entry 4). ${ }^{1} \mathrm{H} \mathrm{NMR}\left(300 \mathrm{MHz}, \mathrm{CDCl}_{3}\right) \delta$ : $3.76(3 \mathrm{H}, \mathrm{s}$, COOMe), $4.65\left(2 \mathrm{H}, \mathrm{s}, \mathrm{CH}_{2}\right), 6.21(2 \mathrm{H}, \mathrm{t}, \mathrm{J}=2.1 \mathrm{~Hz}, \mathrm{HCCH}), 6.67(2 \mathrm{H}, \mathrm{t}, \mathrm{J}=2.1 \mathrm{~Hz}, \mathrm{HCNCH})$. ${ }^{13} \mathrm{C}$ NMR $\left(75 \mathrm{MHz}, \mathrm{CDCl}_{3}\right) \delta: 50.81\left(\mathrm{CH}_{3}\right), 52.62\left(\mathrm{CH}_{2}\right), 109.22(\mathrm{HCCH}), 121.88(\mathrm{HCNCH})$, $169.33(\mathrm{C}=\mathrm{O}) . \mathrm{IR}\left(\mathrm{cm}^{-1}\right) v_{\max }$ : 1755 (br. $\left.\mathrm{C}=\mathrm{O}\right)$. MS: m/z (\%): $139\left(\mathrm{M}^{+}, 60\right), 80(100), 53$ (17). 
Diethyl 1H-pyrrol-1-ylmethylphosphonate ${ }^{10}$ (entry 5). ${ }^{1} \mathrm{H}$ NMR $\left(300 \mathrm{MHz}, \mathrm{CDCl}_{3}\right) \delta: 1.26$ $\left(3 \mathrm{H}, \mathrm{d}, \mathrm{J}=6.6 \mathrm{~Hz}, \mathrm{CH}_{3}\right), 1.27\left(3 \mathrm{H}, \mathrm{d}, \mathrm{J}=6.6 \mathrm{~Hz}, \mathrm{CH}_{3}\right), 3.96-4.09\left(4 \mathrm{H}, \mathrm{m}, \mathrm{P}(\mathrm{O})\left(\mathrm{OCH}_{2} \mathrm{CH}_{3}\right)_{2}, 4.26\right.$ $\left(2 \mathrm{H}, \mathrm{d}, \mathrm{J}=11.6 \mathrm{~Hz}, \mathrm{NCH}_{2} \mathrm{P}\right), 6.17(2 \mathrm{H}, \mathrm{dt}, \mathrm{J}=0.8 \mathrm{~Hz}, \mathrm{~J}=2.2 \mathrm{~Hz}, \mathrm{HCCH}), 6.72(2 \mathrm{H}, \mathrm{dt}, \mathrm{J}=0.6 \mathrm{~Hz}$, $\mathrm{J}=2.2 \mathrm{~Hz}, \mathrm{HCNCH}) .{ }^{13} \mathrm{C}$ NMR $\left(75 \mathrm{MHz}, \mathrm{CDCl}_{3}\right) \delta: 16.49\left(\mathrm{~d}, \mathrm{~J}=5.8 \mathrm{~Hz}, \mathrm{CH}_{3}\right), 45.93(\mathrm{~d}, \mathrm{~J}=$ $\left.158.1 \mathrm{~Hz}, \mathrm{NCH}_{2} \mathrm{P}\right), 62.88$ (d, J=6.9 Hz, $\mathrm{P}(\mathrm{O})\left(\mathrm{OCH}_{2} \mathrm{CH}_{3}\right)_{2}, 109.12(\mathrm{HCCH}), 121.97(\mathrm{HCNCH})$. ${ }^{31} \mathrm{P}$ NMR (109 MHz, $\left.\mathrm{CDCl}_{3}\right) \delta$ : 19.99. IR $\left(\mathrm{cm}^{-1}\right) v_{\max }$ : 1497. MS: m/z (\%): $217\left(\mathrm{M}^{+}, 54\right), 202$ (17), 174 (13), 107 (29), 81 (33), 80 (100), 53 (15).

1-(2-Methoxyphenyl)1 $\mathrm{H}$-pyrrole ${ }^{11}$ (entry 6). ${ }^{1} \mathrm{H} \mathrm{NMR}\left(300 \mathrm{MHz}, \mathrm{CDCl}_{3}\right) \delta: 3.83\left(3 \mathrm{H}, \mathrm{s}, \mathrm{CH}_{3}\right)$, $6.31(2 \mathrm{H}, \mathrm{t}, \mathrm{J}=2.2 \mathrm{~Hz}, \mathrm{HCCH}), 6.98(2 \mathrm{H}, \mathrm{t}, \mathrm{J}=2.2 \mathrm{~Hz}, \mathrm{HCNCH}), 6.99-7.04\left(2 \mathrm{H}, \mathrm{m}, \mathrm{CH}_{\text {arom }}\right)$, 7.22-7.30 (2H, m, $\left.\mathrm{CH}_{\text {arom. }}\right){ }^{13} \mathrm{C}$ NMR $\left(75 \mathrm{MHz}, \mathrm{CDCl}_{3}\right)$ 8: $55.91\left(\mathrm{OCH}_{3}\right), 108.86(\mathrm{HCCH})$, $112.41\left(\mathrm{CH}_{\text {arom. }}\right), 121.01\left(\mathrm{CH}_{\text {arom }}\right), 122.17(\mathrm{HCNCH}), 125.86\left(\mathrm{CH}_{\text {arom. }}\right), 127.56\left(\mathrm{CH}_{\text {arom }}\right), 130.38$ (C $\left.\mathrm{C}_{\text {arom. }}\right), 152.83$ (C $\left.\mathrm{C}_{\text {arom. }}\right)$. IR ( $\left.\mathrm{cm}^{-1}\right) v_{\max }: 1512,1598 . \mathrm{MS}: \mathrm{m} / \mathrm{z}(\%): 173\left(\mathrm{M}^{+}, 100\right), 172(46), 158$ (13), 144 (14), 130 (13), 115 (15), 77 (14). Commercially available (Ambinter Screening Library).

\section{Acknowledgements}

We thank the BOF (Bijzonder Onderzoeksfonds, Universiteit Gent; Research Fund Ghent University) and the Fonds voor Wetenschappelijk Onderzoek Vlaanderen (Fund for Scientific research Flanders) for financial support of this research.

\section{References}

1. See for example (a) De Kimpe, N.; Abbaspour Tehrani, K.; Stevens, C.; De Cooman, P. Tetrahedron 1997, 53, 3693 and references cited therein. (b) Trofimov, B. A.; Tarasova, O. A.; Mikhaleva, A. I.; Kalinina, N. A.; Sinegovskaya, L. M; Henkelmann, J. Synthesis 2000, 11, 1585. (c) Almerico, A. M.; Montalbano, A.; Diana, P.; Barraja, P.; Lauria, A.; Cirrincione G.; Dattolo, G. ARKIVOC 2001, (vi), 129. (d) Trofimov, B. A.; Markova, M. V.; Morozova, L. V.; Mikhaleva, A. I. ARKIVOC 2001, (ix), 24. (e) Chien, T-C.; Meade, E. A.; Hinkley, J. M.; Townsend, L. B. Org. Lett. 2004, 6, 2857. (f) Ramanathan, B.; Keith, A. J.; Armstrong, D.; Odom, A. L. Org. Lett. 2004, 6, 2957. (g) Trofimov, B. A.; Zaitsev, A. B.; Schmidt, E. Y.; Vasil'tsov, A. M.; Mikhaleva, A. I.; Ushakov, I. A.; Vashchenko, A. V.; Zorina, N. V. Tetrahedron Lett. 2004, 45, 3789.

2. Dieltiens, N.; Stevens, C.V.; De Vos, D.; Allaert, B.; Drozdzak, R.; Verpoort, F. Tetrahedron Lett. 2004, 45, 8995.

3. See for example (a) Bertz, S. H.; Dabbagh, G.; Williams, L. M. J. Org. Chem. 1985, 50, 4415. (b) Padwa, A.; Norman, B. H J. Org. Chem. 1990, 4801. (c) Ezquerra, J.; Pedregal, C.; Rubio, A.; Valenviano, J.; Navio, J. L.G.; Alvarez-Builla, J.; Vaquero, J. J. Tetrahedron Lett. 
1993, 34, 6317. (d) Dell'Erba, C.; Mugnoli, A.; Novi, M.; Pani, M.; Petrillo, G.; Tavani C. Eur. J. Org. Chem. 2000, 903.

4. Kotha, S.; Mandal, K. Tetrahedron Lett. 2004, 45, 2585.

5. Nishiguchi, T.; Kurooka, A.; Fukuzumi, K. J. Org. Chem. 1974, 39, 2403.

6. Clayden, J.; Greeves, N.; Warren, S.; Wothers, P. In Organic Chemistry 1192, Oxford University Press: New York, 2001; p 1512.

7. Commercially available (Aldrich).

8. Baeckvall, J. E.; Nystroem, J. E. J. Chem. Soc., Chem. Comm. 1981, 2, 59.

9. Pittet, A. O.; Pascale, J. V.; Hruza, D. E. Ger. Offen. 1973, 63 pp. DE 2312996, Chem. Abstr. $80,14839$.

10. Gustowski, W.; Kocor, M.; Michalski, J. B. Acad. Pol. Sci-Chim. 1973, 21, 887.

11. Commercially available (Ambinter Screening Library). 